\title{
Prevalence and Types of Disease Management Programs in Community Pharmacies in California
}

\author{
ANANDI V. LAW, PhD; MARK P. OKAMOTO, PharmD; and PETER S. CHANG, PharmD
}

\begin{abstract}
OBJECTIVES: To (1) evaluate the prevalence and types of disease management (DM) programs provided by independent and chain community pharmacies in the state of California, (2) examine the interest among community pharmacists in establishing programs, and (3) assess perceived barriers to developing a successful DM program in community pharmacy.

METHOD: An exploratory telephone survey was conducted from February 2003 to February 2004 to collect data from community (primarily independent and chainstore) pharmacies across California. The survey evaluated existence (or lack of) and types of DM programs in community pharmacies in California. Pharmacies that did not have a DM program were queried on their interest and decisionmaking authority in establishing new programs. Pharmacies that had existing DM programs were sent a follow-up survey to determine the details of the DM programs, including challenges in establishing DM programs, reimbursement issues, and program effectiveness.
\end{abstract}

RESULTS: The sample comprised 1,875 pharmacies, 60 (3.2\%) of which had existing DM programs. There were significantly more independent pharmacies (37) with DM programs than chain-store pharmacies (23), $P<0.001$. There was a statistically significant difference between independent and chain pharmacies in operating hours, number of pharmacist and nonpharmacist staff members per day, and proximity to a clinic or hospital $(P<0.05)$. The most common type of DM program was diabetes, and the second most common type was asthma. Limited time, limited staff, and limited reimbursement were the 3 most commonly reported barriers to establishing new DM programs. About $20 \%$ of the sample that did not have a DM program reported interest in developing DM programs, and an equal percentage reported having the decision-making authority to start a program. There were no differences between independent and chain pharmacies on interest $(P=0.234)$; however, there were significantly more chain pharmacists that did not have the decision-making authority. Of the 18 of 60 pharmacies $(30 \%)$ that had DM

programs and responded to the follow-up survey, 9 respondents (50\%) reported monitoring medications as part of their DM program. Fifteen of $18(83 \%)$ perceived lack of reimbursement as a challenge to implementing DM programs. Only 2 pharmacies reported an increase in revenue as a gain from the program, and 2 reported cost savings. Improved patient satisfaction was reported by 16 of 18 respondents $(89 \%)$ with DM programs, but only $8(44 \%)$ reported that patient satisfaction was being measured.

CONCLUSION: The study found that the prevalence of DM programs was very low among California community pharmacies and the interest in developing these programs moderate, attributable to several barriers such as lack of time, lack of reimbursement, and lack of trained personnel. Some of these barriers could be addressed to encourage the development and proliferation of DM programs that would improve patient outcomes and expand practice roles of pharmacists.

KEYWORDS: Disease management programs, Community pharmacies, Telephone survey, California pharmacies, Community pharmacists

J Manag Care Pharm. 2005;11(6):505-12
$\mathrm{T}$ he profession of pharmacy has been shifting from a practice that is product-focused to a practice that is patient-focused by ensuring that patients receive appropriate drug therapy and by communicating appropriate drug information to improve and promote patient health. One aspect of delivering care that has become increasingly of interest to pharmacists is disease management (DM). The Disease Management Association of America defines DM as "a system of coordinated healthcare interventions and communications for populations with conditions in which patient self-care efforts are significant." $D$ DM often involves identification of at-risk individuals by using a systematic population-based approach, implementing detailed programs of care and evaluating outcomes that are used in the care processes to achieve continuous quality improvement. ${ }^{2}$

A DM program requires an understanding of the progression of a disease and use of targeted interventions at critical points (via evidence-based practices) in order to delay or prevent morbidity and mortality. ${ }^{3}$ Like pharmaceutical care, DM programs are designed to achieve optimal patient outcomes but are directed to a specific patient population and generally focus on one disease. DM uses a systematic approach to attain coordinated, continuous quality of care with the intent to decrease the overall costs of managing a chronic disease. In order to be successful, an effective program also requires a collaborative effort by patients, physicians, and other health care professionals as well as program administration, information management, and financial management.

The diseases typically targeted by DM programs have the following characteristics: (a) prevalent chronic condition, (b) high resource utilization, (c) potential for lifestyle modification

\section{Authors}

ANANDI V. LAW, PhD, is an associate professor and PETER S. CHANG, PharmD, is a research fellow, Department of Social and Administrative Sciences, Western University of Health Sciences, Pomona, California; MARK P. OKAMOTO, PharmD, is director of assessment and an associate professor, Department of Social and Administrative Sciences, and an associate professor of pharmacy practice, Western University of Health Sciences, Pomona, California.

AUTHOR CORRESPONDENCE: Anandi V. Law, PhD, Associate Professor, Department of Social and Administrative Sciences, Western University of Health Sciences, 309 E. Second St., College Plaza, Pomona, CA 91766-1854. Tel: (909) 469-5645; Fax: (909) 469-5428; E-mail: alaw@westernu.edu

Copyright $\odot$ 2005, Academy of Managed Care Pharmacy. All rights reserved. 
to improve outcomes, (d) a variety of treatment options, and (e) a high risk of negative outcomes. ${ }^{3}$ Common examples of disease states targeted by DM programs are asthma, diabetes, hypertension, osteoporosis, and hyperlipidemia. Physicians often cannot provide the necessary attention in managing these chronic conditions because of the limited time available for dealing with acute concerns of the patient. Pharmacists can help fill this gap by providing an interface with the physician and patient in interventions such as self-care education, monitoring intermediate outcomes such as blood pressure or hemoglobin Alc, or drug dosage adjustment under protocol. Pharmacists can utilize their training in patient care and drug therapy management to perform tasks under the aegis of the physician and treatment guidelines in order to provide continuous care and treatment management for patients with chronic conditions.

The community pharmacy is a desirable setting for providing DM for several reasons. People visit pharmacies more often than any other health care facility. 4.5 Community pharmacies account for dispensing the majority of prescriptions in the U.S. (82.7\% of the $\$ 203.1$ billion volume of sales [3.22 billion prescriptions in 2003]). ${ }^{6}$ This accessibility of pharmacists creates the opportunity for patients and pharmacists to develop rapport, which is important in establishing trust and participation in DM programs. Also, prescription databases allow pharmacists to readily retrieve patient-specific information to screen for high-risk patients and identify potential drug-related problems. Furthermore, some pharmacists are currently receiving certificate training in disease management, which increases their ability to monitor and document their services and gives them additional skills to establish well-structured programs. ${ }^{7-9}$

Previous research has helped demonstrate that the clinical services provided by pharmacists reduce the consumption of health care resources, reduce cost, maximize the efficiency of care, and minimize adverse drug reactions. ${ }^{10-14}$ Patient response to these types of services has been positive..$^{15,16}$ Based on the potential for professional satisfaction, favorable impact on clinical outcomes, cost reduction, and increased reimbursement for pharmacists providing these cognitive services, it would appear that DM programs would be common in the community setting. However, a review of the published literature reveals a scarcity of documentation of programs and of outcomes from these programs in community pharmacy settings, although there are some data available from inpatient or hospital outpatient (ambulatory care) settings. ${ }^{17,18}$

There also have been anecdotal reports and abstracts presented at meetings about existing DM programs in the community setting. However, with the exception of a few demonstration projects (for example, project ImPACT and the Asheville Project), there is little evidence of the widespread success of these programs in terms of improved patient outcomes and/or cost effectiveness and/or reimbursement to participating pharmacists. ${ }^{11,19-21}$
Some questions arise from this lack of evidence, including reasons for it, and the verity of the trend, i.e., is the lack of widespread DM programs in community pharmacies real or is it a result of poor documentation? If there are, in fact, few operational DM programs in community pharmacy, what are the reasons for the low prevalence? How can we address any challenges that exist in establishing, maintaining, and achieving success with DM programs? Answers to these questions are important to the profession of pharmacy for future professional development and perhaps for obtaining reimbursement.

These questions have additional significance with the advent of Medicare Part D, in which pharmacists will be part of the Medication Therapy Management Program, providing DM-like services to Medicare beneficiaries who qualify. ${ }^{22}$ A first step to address the questions outlined above would involve examination of the prevalence of DM programs in community pharmacies. Inquiry into interest in starting such programs and the challenges in establishing programs could provide further insight into the future development of DM programs in community pharmacies. Documenting the degree of success that pharmacists have experienced with DM programs, including the magnitude of improvement of patient outcomes and receipt of reimbursement for these services, could serve as a model or template for the future. Knowledge of the challenges or barriers to establishing such programs can stimulate attempts to overcome some of the barriers and facilitate development and proliferation of DM programs.

The objectives of this study were to (1) evaluate the prevalence and types of DM programs currently available among independent and chain community pharmacies in the state of California, (2) examine the interest among pharmacists in establishing programs, and (3) assess perceived barriers to development of a successful DM program. The study also examined operational characteristics of existing DM programs.

\section{Methods}

\section{Study Design}

An exploratory telephone survey design was used to assess the status of DM programs in California community pharmacies and to address the study objectives. This study and the telephone and follow-up surveys were approved by the institutional review board (IRB) of the authors' institution.

\section{Participants and Setting}

A list comprising the names, addresses, and telephone numbers of all community pharmacies (5,270 pharmacies across 1,049 zip codes) in California was obtained from a commercial mailing company (TPS Products and Services, Inc., Newcastle, CA). Each pharmacy was identified as either independent or chain.

\section{Data Collection}

Two surveys were developed-an initial telephone screening 
survey designed to identify pharmacies with DM programs and a follow-up survey to obtain program-specific information from pharmacies that reported DM programs. These surveys were examined for face and content validity by a panel of 3 faculty members and 4 practicing community pharmacists and modified accordingly. In the initial telephone survey, we defined DM for pharmacists as "a formalized evaluation and management program or clinic for different chronic disease states such as hypertension, diabetes mellitus, asthma, high cholesterol, and osteoporosis. This does not include immunization or one-time health fairs." When a more detailed description was provided, a DM program was described as requiring the pharmacist to monitor and manage patients on a periodic basis in order to impact their adherence to lifestyle modification and therapy in an attempt to improve their clinical outcomes.

Data were collected between February 2003 and February 2004 by 9 third-year (P3) doctorate of pharmacy (PharmD) students during their 6-week clerkship rotations. The screening survey was administered via telephone interviews to pharmacies during normal pharmacy business hours. The clerkship students had completed a required P3 course in pharmacy management that included a project on designing a disease management clinic. For consistency in data collection, these students were also trained by the investigators in presenting the telephone script and paraphrasing the definition of disease management to the respondents.

Calls to each pharmacy were based on random assignments presorted by county and zip code. Following a brief introduction, the caller proceeded to ask the respondent at the pharmacy if he or she consented to participate in the study. The respondent could be a staff pharmacist or manager. An initial question was asked to identify the presence or absence of a DM program. Respondents who stated that they did not have an existing DM program in place were asked for possible reasons for not having or not wanting a program in place (perceived barriers). The respondents were also asked about their interest in developing a DM program in the future and about their decision-making authority to establish DM programs. Respondents who stated that they had an existing program were asked a few questions to determine the nature and extent of the program, namely, the types of DM programs and diseases they managed, target patient groups, length of operation, and if any outcomes were measured.

Information that characterized the pharmacies and was considered relevant to presence or absence of the DM programs was obtained from all pharmacies. This information included the type of community pharmacy (to confirm information in our database), the hours of operation, the number of pharmacists and nonpharmacists on staff, type of setting (i.e., urban or rural), and the proximity (within a mile) to a clinic or hospital.

Pharmacies with a DM program were requested to complete a follow-up survey in order to obtain detailed information about the program, including protocols and services performed in the

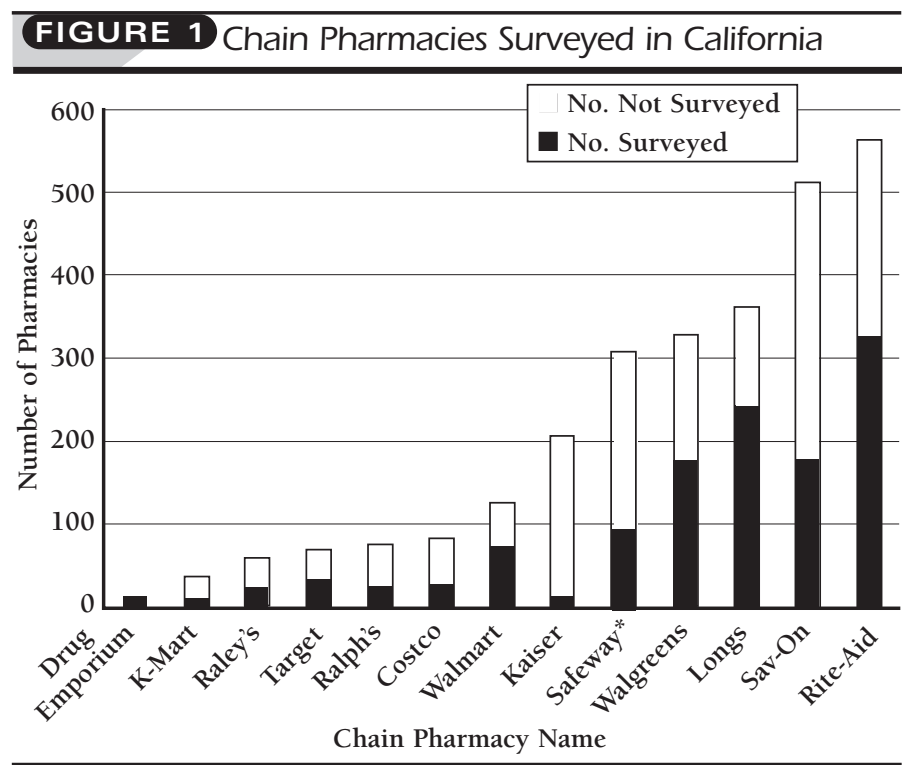

* Safeway Pharmacies included Safeway, VONS, and Pavilions chain-store pharmacies.

management of patients, challenges in instituting the program, outcomes measured, and reimbursement patterns. The follow-up surveys were faxed to pharmacies with DM programs with the option of being returned by either facsimile or mail. Pharmacists had the opportunity to refuse participation at any time.

\section{Data Analysis}

Data were analyzed using the statistical software SPSS for Windows, version 11.0.1. ${ }^{23}$ Descriptive statistics were reported for all variables being measured. The type of pharmacy (independent versus chain) was used as the unit of analysis. Chi-square tests were used to compare the independent and chain pharmacies on nominal variables such as type of setting, proximity to clinic or hospital, DM program currently in place, interest and decision-making authority in developing DM programs, DM program in the past, and ability to develop DM program. Independent sample $t$ tests were used to analyze continuous variables such as the hours of operation, the number of pharmacists on staff, and the number of nonpharmacists on staff. The level of significance was set at an alpha level of 0.05. Descriptive analyses were also conducted on the data from the follow-up survey.

\section{Results}

We attempted to contact a total of 2,069 (39\%) of the 5,270 pharmacies on the list, spanning 670 (64\%) zip codes. Of these, 88 pharmacies could not be reached because of sampling frame error, for example, lack of phone number or disconnected phone numbers with no further information available. Of the remaining $1,981,106$ pharmacies (5.4\%) elected not to participate. Completed responses were obtained from 1,875 


\section{TABLE 1 Characteristics of Chain and Independent Community Pharmacies in California and Prevalence of DM Programs}

\begin{tabular}{|c|c|c|c|}
\hline & $\begin{array}{c}\text { Independent } \\
\text { Pharmacies } \\
(\mathrm{N}=555)\end{array}$ & $\begin{array}{c}\text { Chain } \\
\text { Pharmacies } \\
(\mathrm{N}=1,283)\end{array}$ & $\begin{array}{c}P \\
\text { Value }\end{array}$ \\
\hline $\begin{array}{l}\text { Type of setting (frequency) } \\
\text { Urban } \\
\text { Rural }\end{array}$ & $\begin{array}{r}465 \\
84\end{array}$ & $\begin{array}{r}1,039 \\
243\end{array}$ & 0.96 \\
\hline $\begin{array}{l}\text { Proximity to clinic/hospital (frequency) } \\
\text { Yes } \\
\text { No }\end{array}$ & $\begin{array}{l}346 \\
203\end{array}$ & $\begin{array}{l}888 \\
394\end{array}$ & $<0.05$ \\
\hline Hours open per day (mean \pm SD) & $9.15 \pm 1.52$ & $11.76 \pm 2.79$ & $<0.05$ \\
\hline $\begin{array}{l}\text { Number of pharmacist(s) working } \\
\text { per day }(\text { mean } \pm S D)\end{array}$ & $1.42 \pm 0.70$ & $1.84 \pm 0.82$ & $<0.05$ \\
\hline $\begin{array}{l}\text { Number of nonpharmacist(s) working } \\
\text { per day }(\text { mean } \pm \text { SD) }\end{array}$ & $2.78 \pm 2.33$ & $3.18 \pm 2.03$ & $<0.05$ \\
\hline Existing DM program (frequency) & $37(6.7 \%)$ & $23(1.8 \%)$ & $<0.001$ \\
\hline DM program in the past (frequency) & $6(1.1 \%)$ & $5(0.4 \%)$ & 0.452 \\
\hline $\begin{array}{l}\text { Able to make decisions on establishing } \\
\text { a new DM program (frequency) }\end{array}$ & $313(56.4 \%)$ & 107 (8.3\%) & $<0.001$ \\
\hline $\begin{array}{l}\text { Interested in developing a new DM } \\
\text { program (frequency) }\end{array}$ & $118(21.3 \%)$ & $243(18.9 \%)$ & 0.234 \\
\hline
\end{tabular}

\section{TABLE 2 Perceived Barriers}

to Developing a DM Program

\begin{tabular}{l|c}
\hline Barrier & $\begin{array}{c}\text { Number of Responses } \\
(\mathrm{N}=1,467)\end{array}$ \\
\hline Limited time & $681(46.4 \%)$ \\
\hline Other reasons* & $277(18.9 \%)$ \\
\hline Limited staff & $259(17.7 \%)$ \\
\hline Reimbursement issues & $108(7.4 \%)$ \\
\hline Limited space & $49(3.3 \%)$ \\
\hline High cost & $44(3.0 \%)$ \\
\hline Limited patient acceptance & $37(2.3 \%)$ \\
\hline * Other reasons included corporate philosophy or policy, limited number of patients, \\
and lack of knowledge about disease management (DM).
\end{tabular}

chain, independent, and specialty pharmacies that participated in the telephone surveys, accounting for $36 \%$ of the community pharmacies in California. Our sample had a reasonable distribution across the zip codes in California where pharmacies are present. The sample comprised 1,283 chain-store pharmacies (including traditional chains, grocery store chains, and mass merchandisers), 555 independent pharmacies, and 37 specialty pharmacies. Figure 1 shows the breakdown of the large chain-store pharmacies in the state that we surveyed. Only 60 (3.2\%) of the surveyed pharmacies reported that they had a DM program in place. Of the 60 pharmacies with DM programs, only 18 pharmacies (30\%) completed the comprehensive follow-up survey, 16 reported that they did not want to provide the information, and 26 did not respond despite repeated reminders. The 60 pharmacies were distributed across 55 zip codes.

Table 1 summarizes the demographic information on the sample, using independent and chain pharmacies as the unit of analysis. There was a statistically significant difference between independent and chain pharmacies in terms of operating hours, number of pharmacist and nonpharmacist staff members per day, and proximity to clinic or hospital $(P<0.05)$. Upon examining the data, more chain pharmacies were located near clinics or hospitals, had longer pharmacy business hours, and more nonpharmacist staff members. Table 1 also presents the prevalence of DM programs among the surveyed pharmacies. Independent pharmacies showed a significantly higher prevalence than chains, both in absolute numbers as well as proportionately ( $7 \%$ and $2 \%$, respectively, $P<0.001$ ). Of those that did not currently have DM programs in place, only 6 independent pharmacies and 5 chain pharmacies reported having had a DM program in the past. None of the specialty pharmacies had a DM program in place.

One of the objectives in this study was to assess pharmacist interest in developing a DM program for those pharmacies that did not have a program currently in place. Only 358 of 1,757 pharmacies (approximately 20\%) reported an interest in implementing DM programs in the future. There was no statistically significant difference between the chain pharmacies and independent pharmacies on whether or not they had an interest in developing DM programs $(P=0.234)$. There were significantly more independent pharmacies (61\%) that reported that they had the authority to make decisions on establishing new DM programs as compared with chain pharmacies (9\%); $72 \%$ of the total sample stated that they were restricted in making these decisions.

Table 2 outlines the reasons that those pharmacies did not have or want DM programs (some pharmacies gave multiple reasons). As seen, limited time, limited staff, and reimbursement issues were the top 3 reasons that were perceived barriers to establishing DM programs. Among "other reasons" cited were corporate philosophy or decisions, limited number of patients, and lack of knowledge about DM.

For the 60 pharmacies that responded that they had a DM program in place, Table 3 illustrates the specific DM programs that are being implemented. Diabetes DM programs were the most common type, followed by asthma. It should be noted that some pharmacies incorporated more than one DM program simultaneously.

Of the 18 pharmacies that did have a DM in place and also completed the comprehensive follow-up surveys, 9 were independent and 9 were chain pharmacies. The small sample 
size (18) precluded any meaningful data analysis. Table 4 presents a summary of selected information provided by these pharmacies. The programs commonly included education about the disease state and medications, as well as medication monitoring. Less commonly featured were the initiation and adjustment of drug therapy. Interestingly, the commonly reported challenges in establishing the DM programs coincided with the perceived barriers mentioned by pharmacies without DM programs. Less than half of the programs appeared to measure indicators of program effectiveness (outcomes). Patient satisfaction was the most frequent outcome measure reported as a gain from the program. Sixteen respondents self-reported that patients were satisfied with the DM program although only half the number (8 pharmacies) responded that they had measured patient satisfaction. Only 2 of 18 pharmacies reported an increase in revenue as one of the gains from the DM programs; a similar number reported cost savings (not shown in Table 4).

\section{Discussion}

The results of this study showed that the prevalence of DM programs and interest in developing one were low among community pharmacies in California. There are at least 3 possible interpretations of these findings:

1. The low level of prevalence and interest is perhaps associated with the type of perceived barriers that make it difficult to develop or continue such a program. The literature indicates that pharmacists also perceive the following barriers to performing cognitive services in community pharmacies: excessive workload, lack of privacy, patient attitudes, store layout, interacting with physicians, and cost of implementation. ${ }^{24,25}$ Some of these factors reported by study respondents included budget, lack of time, limited staffing, and, most important, lack of reimbursements. Some of these barriers could be addressed and resolved in order to promote the future development of successful DM programs. (For example, time and staffing issues can be addressed by reallocation of resources and better management). However, reimbursement for pharmacist services presents a more difficult challenge that all pharmacies experience. Existing DM programs report a variety of reimbursement patterns, including direct patient payment and health plan or employer reimbursement for a few programs. Addressing this issue may increase the interest level in developing DM programs. In addition, pharmacies can work with health plans on developing defined reimbursement protocols; once again, there needs to be a body of evidence that pharmacists can reduce system costs and improve outcomes as compared with usual care, and there needs to be a "buy-in" from health plans and employers.

2. Another possible explanation for the low prevalence of DM that we found in California pharmacies is that pharmacists may be providing some of these services and there may be DM programs in existence, but pharmacists have not
TABLE 3 Types of DM Programs Implemented in Independent and Chain Pharmacies

\begin{tabular}{l|c|c|c}
\hline & $\begin{array}{c}\text { Independent } \\
\text { Pharmacy } \\
(\mathbf{N}=37)\end{array}$ & $\begin{array}{c}\text { Chain } \\
\text { Pharmacy } \\
(\mathbf{N}=23)\end{array}$ & $\begin{array}{c}\text { Total } \\
\text { Programs* } \\
(\mathbf{N}=81)\end{array}$ \\
\hline Diabetes & 17 & 12 & $29(36 \%)$ \\
\hline Asthma & 16 & 4 & $20(25 \%)$ \\
\hline Hyperlipidemia & 7 & 6 & $13(16 \%)$ \\
\hline Hypertension & 6 & 2 & $8(10 \%)$ \\
\hline Osteoporosis & 1 & 1 & $0(2 \%)$ \\
\hline Smoking cessation & 0 & 0 & $9(11 \%)$ \\
\hline Other & 8 & 1 & 2 \\
\hline
\end{tabular}

* The number of disease management (DM) programs exceeds the number of pharmacies that responded because some pharmacies had more than one program operating concurrently.

\section{TABLE 4 Characteristics of DM Programs in Community Pharmacies in California}

\begin{tabular}{|c|c|}
\hline Survey Items and Selected Responses* & Number of Pharmacies $\dagger$ \\
\hline $\begin{array}{l}\text { Activities conducted by pharmacists: } \\
\text { Education about disease state and drug therapy } \\
\text { Recommendations to physicians } \\
\text { Monitoring medications }\end{array}$ & $\begin{array}{c}15 \\
12 \\
9\end{array}$ \\
\hline $\begin{array}{l}\text { Challenges faced by the pharmacy in } \\
\text { developing a DM program: } \\
\text { Lack of reimbursement } \\
\text { Lack of time } \\
\text { Lack of staff }\end{array}$ & $\begin{array}{c}15 \\
11 \\
8\end{array}$ \\
\hline $\begin{array}{l}\text { Specific clinical, economic, and/or humanistic } \\
\text { outcomes being measured: } \\
\text { Compliance with therapy and lifestyle modification } \\
\text { Clinical outcomes } \\
\text { Patient satisfaction with the program }\end{array}$ & $\begin{array}{l}9 \\
7 \\
7\end{array}$ \\
\hline $\begin{array}{l}\text { Areas of documented success with } \\
\text { DM program: } \\
\text { Patient's improved awareness of drug } \\
\text { and disease state } \\
\text { Patient's improved compliance with therapy } \\
\text { Increased satisfaction with pharmacy services }\end{array}$ & $\begin{array}{l}10 \\
9 \\
8\end{array}$ \\
\hline $\begin{array}{l}\text { Gains obtained from the DM program: } \\
\text { Improved patient satisfaction } \\
\text { Professional satisfaction } \\
\text { Improved image in the community }\end{array}$ & $\begin{array}{c}16 \\
14 \\
9\end{array}$ \\
\hline \multicolumn{2}{|c|}{$\begin{array}{l}\text { * Only the } 3 \text { most commonly selected response choices for each question are reported } \\
\text { here. } \\
\text { † Respondents were allowed to choose more than one selection. } \\
\text { DM=disease management. }\end{array}$} \\
\hline
\end{tabular}


documented protocols and outcomes of these programs. ${ }^{26}$ Among the pharmacies in our study that had DM programs, there was very little evidence that well-designed protocols existed that would help assess the effectiveness of the programs. Also, most programs in our sample did not indicate that data were being collected to evaluate the outcomes of the programs. In effect, although a large proportion of the respondents ( $>60 \%$ ) reported that they saw gains from the program, less than half of them reported actually measuring any outcomes. Previous reviews of studies evaluating clinical pharmacy services found similar results and have encouraged the creation of programs with better documentation and methodology for evaluation of outcomes. ${ }^{27,28}$

3. Related to the reasoning in point 2 above is that pharmacists have been slow to advertise/publish the existence or outcomes from these programs. Even among the small sample that had DM programs in our study, there appeared to be reluctance to respond to the follow-up survey; this nonresponse could be attributable to corporate unwillingness to respond to details about the programs, a possible unwillingness to share results of unstructured programs or services, or simply a lack of time for pharmacists to fill out the survey.

The results also showed that independent pharmacies had a higher percentage of DM programs than chain pharmacies. This finding could be attributed to a greater degree of freedom in pharmacy operations management compared with the corporate infrastructure associated with chain pharmacies. This explanation is further strengthened by the finding that a significantly higher proportion of independent pharmacies reported being able to make decisions on establishing new DM programs. This level of autonomy allows independent pharmacies to establish their own budget and time to create a DM and implement it successfully. Further, independent pharmacies may also use DM as a competitive service advantage over chain pharmacies. However, if chain pharmacies decide to expand or diversify their services in this area, it can result in a multifold increase in pharmacies with DM programs, thus greatly impacting the prevalence of such programs in California.

Although the results from this study indicate a low prevalence of DM programs in community pharmacies in California, there are several factors that bode well for community-based DM and for the profession. There is a growing recognition within the profession of the need to document clinical, economic, and humanistic outcomes based on valid and reliable data from well-designed DM protocols in community practice. According to results of an American Pharmacists Association Foundation study in 2004 involving 80 community pharmacy providers in 5 states, pharmacist-directed self-management in patients with diabetes produced improved clinical indicators, higher rates of self-management goal setting and achievement, and increased satisfaction with diabetes care. ${ }^{21}$ This program may serve as a template for community pharmacy to establish, monitor, and document DM.

Further, collaborative practice arrangements between physicians and pharmacists, also known as collaborative drug therapy management or CDTM, provide a conducive environment for growth of DM and is permitted by legislation or regulation in most states. ${ }^{29}$ Pharmacists can collaborate with physicians on mutually agreed-upon protocols in order to improve patient outcomes. CDTM has been used in the management of asthma, diabetes, smoking cessation, and, more recently, emergency contraception. ${ }^{30-32}$ While the term "collaborative practice" does not appear explicitly in the California pharmacy practice regulations, the California Business and Professions Code allows trained pharmacists to provide services ranging from administering injections; ordering or performing routine drug therapy-related patient assessment procedures, including measurement of patient temperature, pulse, and respiration; ordering laboratory tests; or initiating and adjusting drug regimens (including up to a 72-hour supply of a controlled substance, including Schedule II medications) using protocols developed by health professionals and approved by the facility. 33,34

Finally, the passage of the Medicare Prescription Drug, Improvement, and Modernization Act of 2003 (MMA) created a new benefit with coverage for drug products and medication therapy management (MTM) services that may result in greater pharmacist involvement in DM programs..$^{35}$ Although the details of MTM are still under development, MMA may present even more opportunity for pharmacists to become more involved in direct patient care and to influence patient outcomes.

\section{Limitations}

Several limitations should be taken into consideration regarding this study. While this research project attempted to account for all DM programs in community pharmacies in California, only $40 \%$ of all existing pharmacies in the state could be contacted, due to resource constraints. We found during data collection that the definition of DM as perceived by the pharmacists was not consistent; some of the pharmacists contacted did not know what a DM program was, and some pharmacists considered health fairs or annual flu shots and/or certain disease screenings to be DM programs. About 30 respondents who were in this category expressed an interest in more information on these programs for the purpose of establishing DM programs in the future. Thus, lack of knowledge or awareness of DM may also be another factor that could be addressed to increase the impact of DM on population health.

A few of the pharmacies contacted did not have regular staff pharmacists available at the time of interview so the responses collected from the relief pharmacists may not reflect accurate demographics of those pharmacies. Many of the pharmacies contacted were very busy, had a limited number of pharmacists on staff, and were therefore reluctant to answer questions. 
It was also noted that some of the pharmacists who were contacted (mostly chain pharmacists) were prohibited from answering any telephone surveys. Finally, the low response rates for those pharmacies with DM in place, despite an average of 3 follow-up calls, made it impossible to perform inferential analyses regarding the characteristics of the community pharmacy DM programs.

This study was a first attempt to document the prevalence of community pharmacy DM programs in the state of California. The generalizability of the results to the rest of the country may be limited. Future studies should involve surveys of DM programs in community pharmacies in other states to compare the prevalence and the extent of services provided via DM and also to investigate the factors that contribute to the success or failure of community pharmacy DM programs and services.

\section{Conclusion}

Although community pharmacist involvement in disease management has large potential to extend care beyond the physician's office to include services such as therapeutic monitoring, reinforced education about disease states and selfcare, and monitoring of drug therapy and compliance with lifestyle modification, our study found that this form of health care was not delivered widely in community pharmacies in California in 2003-2004.

\section{ACKNOWLEDGMENTS}

The authors wish to thank the pharmacy clerkship students who were instrumental in data collection and Susan Robles, administrative assistant, Western University of Health Sciences, Pomona, CA, for her administrative and data entry support.

\section{DISCLOSURES}

No outside funding supported this research. The results were presented in part as a work-in-progress poster at the American Pharmacists Association Annual Meeting on March 28, 2004, Seattle, WA. The authors disclose no potential bias or conflict of interest relating to this article.

Author Anandi V. Law served as principal author of the study. Study concept and design were contributed by Law and author Mark P. Okamoto. Analysis and interpretation of data were contributed by Law, Okamoto, and author Peter S. Chang. Drafting of the manuscript was the work of Law and Chang, and its critical revision was the work of Law and Okamoto. Statistical expertise was contributed by Okamoto and Law.

\section{REFERENCES}

1. Disease Management Association of America. Definition of disease management Available at: http://www.dmaa.org/definition.html. Accessed March 22, 2005.

2. Epstein RS, Sherwood LM. From outcomes research to disease management: a guide for the perplexed. Ann Intern Med. 1996;124:882-37.

3. Gurnee MC, DaSilva RV. Constructing disease management programs. Manag Care. June 1997. Available at: http://www.managedcaremag.com/ archives/9706/9706.disease_man.shtml. Accessed June 10, 2001.

4. Facts about pharmacists and pharmacies. Available at: http://www.aacp.org/ site/tertiary.asp?TRACKID $=\& V I D=2 \& C I D=686 \& D I D=6340$. Accessed June 9, 2004.
5. Holdford D, Kennedy DT, Bernadella P, Small RE. Implementing disease management on community pharmacy practice. Clin Ther. 1998;20(2):328-39.

6. Levy S. Chain industry is alive and well: NACDS profile. Drug Top. October 11, 2004. Available at: http://www.drugtopics.com/drugtopics/article/ articleDetail.jsp?id=127974. Accessed January 18, 2005

7. Kirsche ML. Pharmacist's role shifts to caregiver as diabetes education efforts advance—chain pharmacy. Drug Store News. October 10, 2003. Available at: http://articles.findarticles.com/p/articles/mi_m3374/is_14_25/ai_ 109181276. Accessed June 9, 2004.

8. National Institute for Standards in Pharmacist Credentialing (NISPC) page. Available at: http://www.nispcnet.org/pressreleases/dyslipidemia_3_03.html. Accessed June 9, 2004.

9. National Institute for Pharmacist Care Outcomes (NIPCO) page. Available at: http://www.ncpanet.org/news_press/press_releases/2003/more_than_500_ pharmacists_achieve_nipco_10-18-2003.shtml. Accessed June 9, 2004.

10. Nola KM, Gourley DR, Portner TS, et al. Clinical and humanistic outcomes of a lipid management program in the community pharmacy setting. J Am Pharm Assoc. 2000;40(2):143-44.

11. Bluml BM, McKenney JM, Cziraky MJ. Pharmaceutical care services and results in project ImPACT: hyperlipidemia. J Am Pharm Assoc. 2000;40(2):157-65.

12. Berringer R, Shibley MC, Cary CC, Pugh CB, Powers PA, Rafi JA. Outcomes of a community pharmacy-based diabetes monitoring program. J Am Pharm Assoc. 1999;39(6):791-97.

13. Munroe WP, Kunz K, Dalmady-Israel C, Potter L, Schonfeld WH. Economic evaluation of pharmacist involvement in disease management in community pharmacy setting. Clin Ther. 1997;19(1):113-23.

14. Rupp MT, McCallian DJ, Sheth KK. Developing and marketing a community pharmacy-based asthma management program. J Am Pharm Assoc. 1997; NS37(6): 694-99.

15. Kradjan WA, Schulz R, Christensen DB, et al. Patients' perceived benefit from and satisfaction with asthma-related pharmacy services. J Am Pharm Assoc. 1999;39(5):658-66.

16. Liu MY, Jennings JP, Samuelson WM, Sullivan CA, Veltri JC. Asthma patients' satisfaction with the frequency and content of pharmacist counseling. J Am Pharm Assoc. 1999;39(4):493-98.

17. Beney J, Bero LA, Bond C. Expanding the roles of outpatient pharmacists: effects on health services utilisation, costs and patient outcomes (Cochrane Review). In: The Cochrane Library, Issue 4; 2001.

18. Singhal PK, Raisch DW, Gupchup GV. The impact of pharmaceutical services in community and ambulatory care settings: evidence and recommendations for future research. Ann Pharmacother. 1999;33(12):1336-55.

19. Cranor CW, Christensen DB. The Asheville Project: short-term outcomes of a community pharmacy diabetes care program. J Am Pharm Assoc. 2003; 43(2):149-59.

20. Cranor CW, Bunting BA, Christensen DB. The Asheville Project: long-term clinical and economic outcomes of a community pharmacy diabetes care program. J Am Pharm Assoc. 2003;43(2):173-84.

21. Garrett DG, Bluml BM. Patient self-management program for diabetes: first-year clinical, humanistic, and economic outcomes. J Am Pharm Assoc. 2005;45(2):130-37.

22. Curtiss FR. Medication therapy management programs-will patient need be satisfied? [editorial]. J Manag Care Pharm. 2005;11(4):352-53.

23. SPSS, version 11.0.1, 2004. SPSS Inc., Chicago, IL.

24. Raisch DW. Barriers to providing cognitive services. Am Pharm. December 1993;NS33(12):54-58.

25. Norwood GJ, Sleath BL, Caiola SM, Lien T. Costs of implementing pharmaceutical care in community pharmacies. J Am Pharm Assoc. 1998;38(6): 755-61.

26. Grainger-Rousseau TJ, Miralles MA, Hepler CD, Segal R, Doty RE, Ben-Joseph R. J Am Pharm Assoc. 1997;NS37(6):647-61. 
27. Schumock GT, Meek PD, Ploetz PA, Vermeulen LC, for the Publications Committee of the American College of Clinical Pharmacy. Economic evaluations of clinical pharmacy-1988-1995. Pharmacotherapy. 1996;16(6):1188-1208.

28. Schumock GT, Butler MG, Meek PD, Vermeulen LC, Arondekar BV, Bauman JL, for the 2002 Task Force on Economic Evaluation of Clinical Pharmacy Services of the American College of Clinical Pharmacy. Evidence of the economic benefit of clinical pharmacy services: 1996-2000.

Pharmacotherapy. 2003;23(1):113-32.

29. Collaborative Practice Agreements. Available at: http: www.go2ec.org/ CollabPracticeAgreements.htm. Accessed May 1, 2005.

30. Kuo GM, Buckley TE, Fitzsimmons DS, Steinbauer JR. Collaborative drug therapy management services and reimbursement in a family medicine clinic. Am J Health Syst Pharm. 2004;61(4):343-54.

31. Punekar Y, Lin SW, Thomas J, III. Progress of pharmacist collaborative practice: status of state laws and regulations and perceived impact of collaborative practice. J Am Pharm Assoc. 2003:43(4):503-10.
32. Irons BK, Lenz RJ, Anderson SL, Wharton BL, Habeger B, Anderson HG, Jr. A retrospective cohort analysis of the clinical effectiveness of a physician-pharmacist collaborative drug therapy management diabetes clinic.

Pharmacotherapy. 2002;22(10):1294-1300.

33. Anonymous. 2005 lawbook for pharmacy. California Board of Pharmacy. Available at: http://www.pharmacy.ca.gov/laws_regs/lawbook.pdf. Accessed May 22, 2005.

34. Hammond RW, Schwartz AH, Campbell MJ, et al. Collaborative drug therapy management by pharmacists--2003. American Colleges of Clinical Pharmacy position statement. Pharmacotherapy. 2003;23(9):1210-25.

35. Passage of the Medicare Prescription Drug, Improvement, and Modernization Act of 2003. November 25, 2002. Available at: www.aphanet.org. Accessed June 20, 2004. 